\title{
Electromagnetically induced transparency in paraffin-coated vapor cells
}

\author{
M. Klein, ${ }^{1,2}$ M. Hohensee, ${ }^{1,2, *}$ D. F. Phillips, ${ }^{1}$ and R. L. Walsworth ${ }^{1,2}$ \\ ${ }^{1}$ Harvard-Smithsonian Center for Astrophysics, Cambridge, Massachusetts 02138, USA \\ ${ }^{2}$ Department of Physics, Harvard University, Cambridge, Massachusetts 02138, USA
}

(Received 14 September 2010; published 31 January 2011)

\begin{abstract}
Antirelaxation coatings in atomic vapor cells allow ground-state coherent spin states to survive many collisions with the cell walls. This reduction in the ground-state decoherence rate gives rise to ultranarrow-bandwidth features in electromagnetically induced transparency (EIT) spectra, which can form the basis of, for example, long-time scale slow and stored light, sensitive magnetometers, and precise frequency standards. Here we study, both experimentally and theoretically, how Zeeman EIT contrast and width in paraffin-coated rubidium vapor cells are determined by cell and laser-beam geometry, laser intensity, and atomic density. Using a picture of Ramsey pulse sequences, where atoms alternately spend "bright" and "dark" time intervals inside and outside the laser beam, we explain the behavior of EIT features in coated cells, highlighting their unique characteristics and potential applications.
\end{abstract}

DOI: 10.1103/PhysRevA.83.013826

PACS number(s): 42.50.Gy, 32.70.Jz, 42.50.Md

\section{INTRODUCTION}

In this article we investigate electromagnetically induced transparency (EIT) [1,2] in wall-coated rubidium vapor cells. Vapor cells with antirelaxation coated walls and no buffer gas [3-6] allow atoms to move ballistically and to preserve their ground-state coherence during wall collisions. Coherent interactions with optical fields over many laser-beam crossings result in very narrow resonances [see Fig. 1(a)]. Coherence lifetimes of up to 1 second have been observed in coated cells $[7,8]$, improving performance of applications such as magnetometry [9], atomic clocks [4], squeezing [10], and quantum information storage [11]. EIT in coated cells has been exploited, for example in slow-light applications [12-14]; here we characterize and compare to a straightforward model the dependence of coated-cell EIT on specific experimental parameters including laser-beam properties and cell temperature. Insights from these measurements and analysis point toward further interesting applications of coated-cell EIT systems.

Two limits are typically exploited with coated cells: (i) high laser intensity such that optical pumping is faster than the beam-crossing time, where coated cells behave much like standard vacuum cells as coherence preserved during wall collisions is quickly eliminated by the laser field as the atom re-enters the beam; and (ii) low laser intensity where velocity averaging during multiple passes of atoms through the beam places the system in a regime analogous to Dicke narrowing [15].

Between the high-intensity, high-bandwidth and lowintensity, Dicke-narrowed limits lies an intermediate regime in which both large bandwidth and effective optical coupling to the atomic coherence state can be found. For example, operating in this intermediate intensity regime, we recently demonstrated a "slow-light beam splitter" [14]. As we show in our present investigation, this regime supports high-contrast EIT, which, along with a long coherence lifetime, is important in light storage [12,16-20] and many other quantum optics

\footnotetext{
*Currently at Department of Physics, University of California, Berkeley, California 94720, USA
}
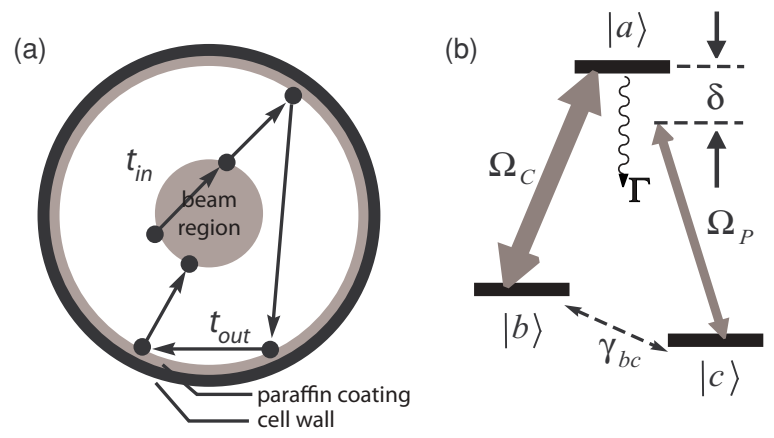

FIG. 1. (a) Cross-sectional view showing the coated vapor cell and beam geometry. Inner filled circle is the laser cross section and outer, open circle indicates the coated vapor cell. Both the bright time interacting with the beam, $t_{\text {in }}$, and the dark time outside it, $t_{\text {out }}$, are important in determining the character of the EIT line shape. (b) Three-level atomic $\Lambda$ system. A strong control field couples the $|b\rangle$ and $|a\rangle$ states with Rabi frequency $\Omega_{c}$ while a weak probe field of Rabi frequency $\Omega_{P}$ couples states $|c\rangle$ and $|a\rangle$. The excited-state decay rate is $\Gamma$.

applications. Here we study and characterize Zeeman EIT in a paraffin-coated, warm ${ }^{87} \mathrm{Rb}$ vapor cell in all three intensity regimes. We observe a characteristic dual-structured EIT line shape, unique saturation limits, and distinct beamcell geometry dependence between the two structures. In a spirit akin to past treatments of buffer gas vapor cells with multistructured, non-Lorentzian EIT line shapes [21-23], we model the system as undergoing a distribution of Ramsey pulse sequences $[24,25]$ inside and outside the laser beam [see Fig. 1(a)]. The ballistic transport mechanism in coated cells is distinct from the diffusive behavior in buffer gas cells, and requires a modified approach. Finally, we propose stored-light experiments that could support high-bandwidth signal storage with long storage times.

The article is organized as follows. In Sec. II we describe the dual-structured nature of EIT in coated cells, drawing on a Ramsey pulse sequence of times in and out of the beam, and outline a numerical model which predicts EIT linewidths. We discuss the coated $\mathrm{Rb}$ vapor cell and experimental apparatus 
in Sec. III. Section IV contains our measurements and comparisons with models, including linewidths (Sec. IV A) and contrasts (Sec. IV B) of the broad and narrow EIT structures and their optimization in the intermediate intensity regime. We also discuss limitations, including radiation trapping and optical pumping into nonparticipating trapped states. We conclude in Sec. $\mathrm{V}$ with a proposed experiment to store a light pulse at high intensity and bandwidth, then retrieve it after a long storage time enabled by the antirelaxation wall coating. In Appendix A, we present details of our numerical model.

\section{THEORY FOR ELECTROMAGNETICALLY INDUCED TRANSPARENCY IN COATED CELLS}

Coated vapor cells are typically used in the limit of low or high laser intensity, resulting in EIT transmission peaks with simple line shapes. Such line shapes are parametrized by a loss rate of atoms, and thus coherence, from the interaction region. In the more general case, for moderate laser-beam size (also see the discussion in Sec. IV A), repeated interactions of coherent atoms with the laser beam lead to EIT line shapes with a dual structure consisting of (i) a broad feature tied to the transit time of atoms moving through the laser beam, and (ii) a narrow feature resulting from atoms repeatedly passing through the beam and then evolving in the dark between wall collisions. A similar situation arises in uncoated vapor cells with buffer gas, with diffusion of atoms out of and back into the laser beam leading to non-Lorentzian shapes [21-23]; in the ballistic motion coated-cell situation here, the times spent inside and outside the laser beam are more sharply defined, thus a clear dual structure emerges.

We model rubidium atoms in a coated cell as three-level $\Lambda$ systems, as shown in Fig. 1(b). A control field (labeled by its Rabi frequency $\Omega_{c}$ ) drives the transition $|b\rangle \rightarrow|a\rangle$, and a weaker probe field $\left(\Omega_{p}\right)$ couples the $|c\rangle \rightarrow|a\rangle$ transition. Initially, these fields optically pump the three-level system into a dark state [26,27], a coherent superposition of $|b\rangle$ and $|c\rangle$ with the same relative phase as $\Omega_{c}$ and $\Omega_{p}:|D\rangle=\left[\Omega_{p}|b\rangle-\right.$ $\left.\Omega_{c}|c\rangle\right] / \Omega$, where $\Omega=\left[\Omega_{c}^{2}+\Omega_{p}^{2}\right]^{1 / 2}$. Atoms in this dark state, which is decoupled from the two input light fields, absorb no light, and transmission of the probe field is maximized. The two-photon detuning is

$$
\delta=\left(v_{c}-v_{p}\right)-v_{b c},
$$

where $v_{c}$ and $v_{p}$ are the respective optical frequencies of the control and probe fields and $v_{b c}$ is the energy difference of the two ground states $(|b\rangle$ and $|c\rangle)$ divided by Planck's constant. On two-photon resonance $(\delta=0)$, the phase relation between the ground states relative to the optical fields is constant. Away from resonance, the atoms accumulate phase relative to the fields at a rate proportional to $\delta$, thus atoms prepared in the dark state evolve into the bright state at a rate of $\sim \delta$. When coherent atoms leave the light fields and return with their coherence intact, atoms can dephase out of $|D\rangle$ even for small $\delta$ values, and transmission becomes a very sensitive function of two-photon detuning.

Three time scales are present in a buffer-gas-free wallcoated vapor cell. As illustrated in Fig. 1(a), an atom moving ballistically across the cell spends (i) a "bright" time $t_{\text {in }}$ interacting with the beam, followed by (ii) a "dark" time $t_{\text {out }}$ outside the beam during which it has at least one interaction with the coated-cell wall before re-entering the beam. During wall collisions, an atom's internal state is preserved while its velocity is randomized; the number of wall collisions between interactions with the beam depends on the ratio of the laser-beam and vapor-cell diameters. The times in and out of the beam are thus both geometry-dependent distributions. (We denote their averages as $\tau_{\text {in }}=\left\langle t_{\text {in }}\right\rangle$ and $\tau_{\text {out }}=\left\langle t_{\text {out }}\right\rangle$.) While in the laser beam at moderate intensity, atoms are optically pumped into the dark state at the rate $|\Omega|^{2} / \gamma_{D}$ (where $\gamma_{D}$ is the Doppler linewidth) and exit the beam at the rate $1 / \tau_{\text {in }}$. The effective dark time can then be extended due to beam crossings for which an atom has a sufficiently large Doppler shift that it does not interact with the laser beam. In paraffin-coated cells, atoms are depolarized only after many collisions with the cell walls. Thus, the remaining time scale (iii) is the inverse of the fundamental decoherence rate $\left(\gamma_{b c}^{-1}\right)$, set by collisions with the coated-cell wall or residual magnetic gradients and is independent of properties of the beam diameter and intensity.

In the experiments presented here, ground-state relaxation from wall collisions and magnetic field gradients is generally small enough that the coherence lifetime is limited by optical pumping: atoms will be re-pumped into the dark state $|D\rangle$ before they would otherwise dephase into the bright state during time spent outside the beam. The pumping rate is determined by both the light field intensity and the size of the beam (which sets the interaction time).

The bright and dark time scales lead coated-cell EIT line shapes to depend sensitively upon laser intensity. For low laser intensities such that $|\Omega|^{2} / \gamma_{D} \ll 1 / \tau_{\text {in }}$, atoms travel through the beam many times before being optically pumped into the dark state. This situation is analogous to Dicke narrowing [15]: the EIT line shape is formed by velocity-averaged atoms that are effectively at rest, with a field interaction based on the average times spent inside and outside the beam. At high laser intensity atoms are pumped into the dark state on time scales short compared to the beam crossing time, and the coating plays a minimal role in determining the line shape. Highintensity line shapes can be modeled as a noninteracting series of velocity classes with varying one-photon Doppler shifts. At moderate intensities, EIT line shapes exhibit both low and high laser intensity behavior simultaneously, and more careful consideration is required.

Repeated interactions of atoms with the light fields are equivalent to a sequence of randomly spaced Ramsey pulses [22-25] in which the two optical fields are turned on and off. During evolution in the dark between "pulses" (beam crossings), atoms undergo coherent phase evolution. Upon returning to the beam, the ensemble average over the distribution for $t_{\text {out }}$ leads to narrow EIT line shapes. In Appendix A we model the coated cell as a collision-free alkali vapor in an infinite cylindrical cell undergoing a distribution of Ramsey sequences (see also [28,29]).

The effect on the EIT line shape of the ends of the cylinder, ignored in the analysis presented in Appendix A, is to decrease the average amount of time the atoms spend in the dark and interact with the beam. Because the beam diameter is smaller than the diameter of the cell, this reduction is most significant for estimating the amount of time atoms spend in 
the dark. The axial velocity distribution and the associated Doppler shifts are included as in [30]. In our infinite cylinder model, an atom's position and velocity are independent, and beam and cell geometry are reduced to bright and dark time distributions. We derive transfer functions that map the initial state of the atomic ensemble to that attained after interaction with the beam or after evolving in the dark. We then iterate these transfer functions to obtain a steady-state solution. This solution corresponds to the state of an ensemble composed of atoms that have independently experienced an infinite Ramsey pulse sequence, where the alternating interaction and phase accumulation periods in each sequence are randomly drawn from the appropriate distribution of times in and out of the laser beam. Results from our model are compared to experimental measurements in Sec. IV A.

\section{EXPERIMENTAL SETUP}

We measured Zeeman EIT in warm ${ }^{87} \mathrm{Rb}$ vapor; the relevant energy levels and coupling laser fields are shown in Figure 2(a). The experimental apparatus [Fig. 2(b)] used an external cavity diode laser tuned to the $D_{1}$ line, operating on the $F=2 \rightarrow F^{\prime}=1$ transition near $795 \mathrm{~nm}$. The laser intensity was regulated with an acousto-optic modulator (AOM), and the spatial profile of the beam cleaned with a pinhole filter, after which beam size was selected by using interchangeable telescope lenses (not shown), with a $1 / e^{2}$ diameter ranging between 1 and $8 \mathrm{~mm}$. We used

(a)

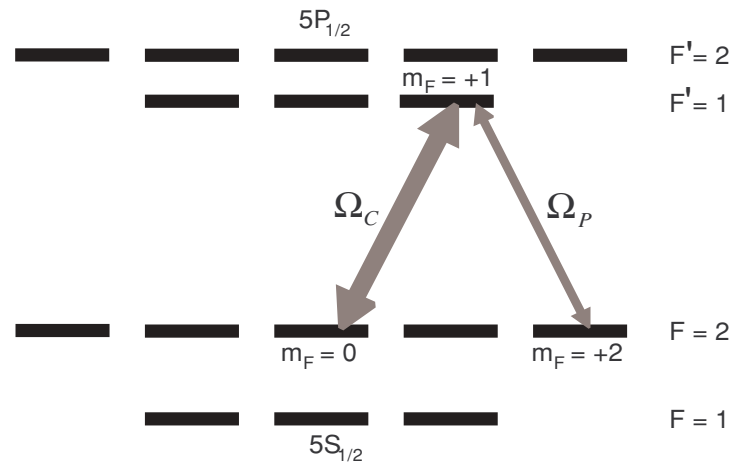

(b)

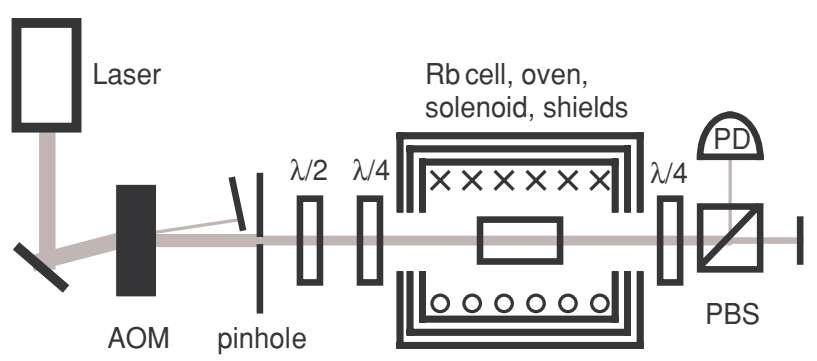

FIG. 2. (a) Level diagram for ${ }^{87} \mathrm{Rb}$, showing the dominant coupling fields for Zeeman EIT (the two other $\Lambda$ systems on the $F=$ $2 \rightarrow F^{\prime}=1$ transition are not shown). (b) Schematic of apparatus used in coated-cell EIT experiments with an acousto-optic modulator $(\mathrm{AOM})$, half- and quarter-waveplates $(\lambda / 2$ and $\lambda / 4)$, polarizing beam splitter (PBS), and photodetector (PD). See text for details. circularly polarized light to couple atomic transitions, so quarter-waveplates $(\lambda / 4)$ on both sides of the Rb cell converted polarization from linear to circular and back. Polarization was rotated by a half-waveplate $(\lambda / 2)$, such that the orthogonally polarized control and probe fields had a 10:1 intensity ratio. The output fields were divided using a polarizing beam splitter (PBS) and the probe field was measured with a photodetector (PD).

The coated $\mathrm{Rb}$ cell was housed inside a plastic oven made of Nylatron ( $\mathrm{MoS}_{2}$-filled nylon). The oven was heated with blown warm air, which in turn conductively heated the cell walls. This allowed uniform cell heating while minimizing mechanical vibrations and eliminating unwanted magnetic field gradients introduced by resistive heaters. In measurements presented here, the cell temperature was varied between 42 and $73{ }^{\circ} \mathrm{C}$, or atomic number density between $7 \times 10^{10}$ and $1 \times 10^{12} \mathrm{~cm}^{-3}$. Cells cooled below this range yielded very low EIT contrast, while cells heated above it both approached the paraffin melting point and resulted in substantial light field absorption. A solenoid surrounded the oven, and the homogeneous magnetic field was swept slowly to vary the two-photon detuning by splitting the otherwise degenerate Zeeman sublevels. The $B$-field sweep range about zero was at most $360 \mathrm{mG}(500-\mathrm{kHz}$ level shift). Three cylindrical layers of high permeability shields screened out stray laboratory magnetic fields. The resulting probe field transmission during a $B$-field sweep constituted an EIT line-shape measurement.

The vapor cell in these experiments was coated with tetracontane $\left(\mathrm{C}_{40} \mathrm{H}_{82}\right)$, a derivative of paraffin, and filled with natural-abundance rubidium $\left(28 \%{ }^{87} \mathrm{Rb}\right)$ following the techniques described in [13]. The cylindrical cell had length $L=$ $5.1 \mathrm{~cm}$ and diameter $D=2.5 \mathrm{~cm}$. The minimum two-photon, EIT resonance width observed was $22 \mathrm{~Hz}$ including losses from wall collisions and from magnetic field gradients. The 22-Hz linewidth implies more than 100 coherence-preserving atomic wall collisions.

\section{EXPERIMENTAL RESULTS}

We measured EIT linewidths and contrasts for the coated cell, considering both the broad and narrow line-shape structures. One motivation is to perform light storage experiments in the coated cell in which we couple effectively to the narrow structure and thus obtain very long storage times.

Note that all applications of EIT to quantum information, magnetometry, and clocks benefit from high contrast. Maximal contrast occurs in an intermediate intensity regime in which there is sufficient laser intensity to promote strong light-atom interaction on the desired transition, yet not so strong as to optically pump the sample during a single pass through the laser beam, nor to optically pump a large fraction of the atoms into the $F=1$ ground hyperfine state, a so-called "trapped state" from which atoms do not participate in EIT. In this intermediate regime, both the narrow resonance attributed to atoms repeatedly interacting with the beam and the broad, single-pass resonance are visible, as in Fig. 3. Further discussion of the direct relationship between EIT line-shape contrast levels and slow-light behavior can be found in [31]. 


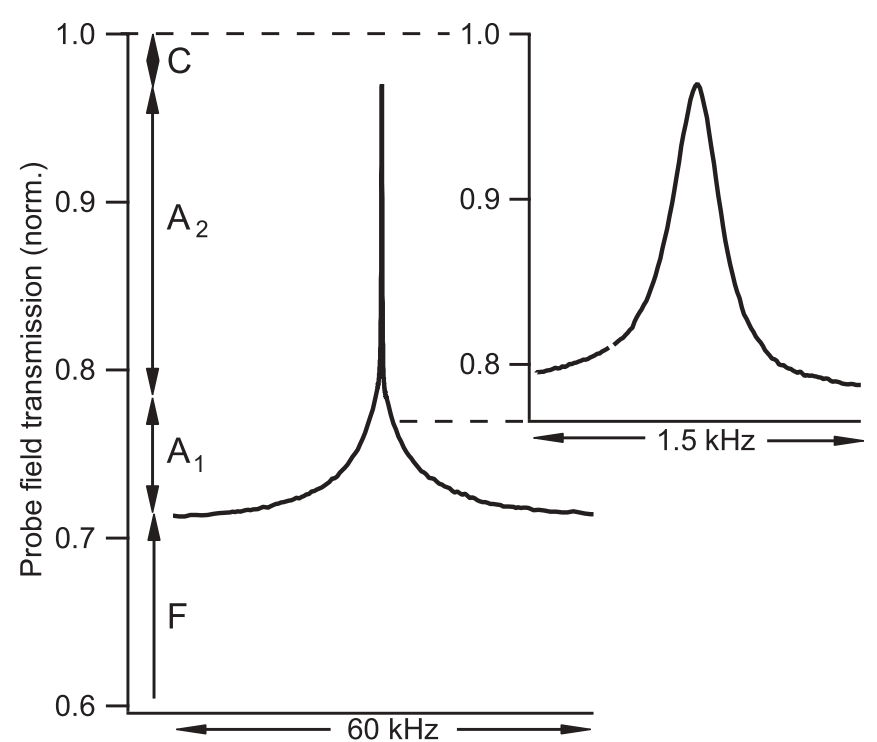

FIG. 3. Measured Zeeman EIT in a warm rubidium vapor cell showing the dual-structure characteristic of coated cells. Probe transmission is plotted against two-photon detuning. Inset shows a zoomed-in version of the narrow structure. Contrast levels are labeled on the left: Floor $(F)$, the off two-photon resonance transmission level measured from zero; amplitudes $A_{1}$ and $A_{2}$ of the broad and narrow structures respectively; and ceiling $(C)$, the difference between the peak of the narrow structure and the maximum probe transmission (transmission far off one- and two-photon resonances), normalized to 1 . Conditions here are $T=42{ }^{\circ} \mathrm{C}, I=2.5 \mathrm{~mW} / \mathrm{cm}^{2}$, and beam diameter $\sim 3 \mathrm{~mm}$. The broad structure has width $16 \mathrm{kHz}$ and the narrow structure has width $260 \mathrm{~Hz}$.

\section{A. Coated-cell EIT linewidths}

EIT linewidth is a key parameter in choosing operating conditions for experiments. For example, magnetometer sensitivity, clock precision, and absolute pulse delay for slow light are determined by the EIT linewidth. As noted earlier, for coated cells there are two widths to consider, as both broad and narrow structures are present in the most general case. Also, linewidth saturation, described below, can limit the choice of operating parameters for the narrow EIT structure: higher laser intensity does not increase the available bandwidth.

The linewidths of both structures in Fig. 3 agree well with the bright-dark time picture described above. The line shape has a broad structure of width $\approx 16 \mathrm{kHz}$, consistent with the transit time of $\tau_{\text {in }}=(d / v)(\pi / 2) \approx 17 \mu \mathrm{s}(d=3 \mathrm{~mm}$ is the beam diameter, $v=280 \mathrm{~m} / \mathrm{s}$ the $\mathrm{rms}$ atomic speed, and $\pi / 2 \mathrm{a}$ geometric factor). The narrow, central peak has a width of 260 $\mathrm{Hz}$, above the lower bound set by measurements of the wall decoherence time allowed in this particular cell $(22 \mathrm{~Hz})$, and below the upper bound set by the estimated average dark time $\tau_{\text {out }}(\sim 375 \mathrm{~Hz})$.

The Ramsey picture of bright and dark times also indicates that the narrow structure linewidth should saturate with increasing intensity, rather than continue power broadening as it would in most vapor-cell systems. This occurs because, for sufficient intensity, light fields optically pump atoms into the dark state in a single pass through the beam. This sets an upper limit on the coherence lifetime: the average time for an atom to leave the beam and return once equals $\tau_{\text {out }}$.

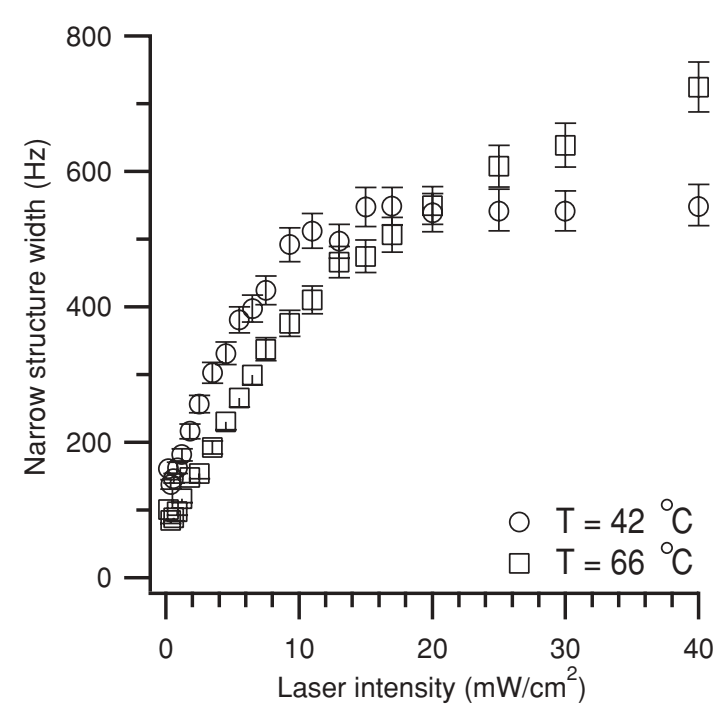

FIG. 4. EIT narrow structure linewidth versus laser intensity for $d=3 \mathrm{~mm}$ at two temperatures. Broad structure widths (not shown) have the standard linear dependence on intensity due to power broadening (see text).

This saturation of the narrow linewidth is confirmed in Fig. 4. At low laser intensity, EIT linewidths exhibit the standard linear power broadening and density narrowing. The widths of both broad and narrow structures increase linearly with laser intensity, scaling like $\gamma_{\text {EIT }} \sim I / \sqrt{N}$, with $I$ the intensity and $N$ the atomic density. Data for $T=51,58$, and $73{ }^{\circ} \mathrm{C}$ are not displayed, but also follow this relationship. Data for a $d=8$-mm beam (not shown) up to $7 \mathrm{~mW} / \mathrm{cm}^{2}$ for our full range of temperatures also obeyed this relationship. At higher intensity, the slopes change and the widths saturate, corresponding to an estimated $\tau_{\text {out }} \sim 1 \mathrm{~ms}$. At the higher temperature shown in Fig. $4\left(T=66^{\circ} \mathrm{C}\right)$, saturation is not yet reached at our maximum laser power. We attribute this effect to control-field depletion at high atomic density, and the resulting variation of the dark state along the length of the cell. A more complex model could incorporate spatial variation of the input light.

Coated vapor-cell experiments can employ a range of input laser-beam sizes (as a fraction of the cell's cross-sectional area). The fraction can be near unity in quantum memory applications [11], where the photon readout probability is maximal when the beam covers the entire area of the atomic ensemble; or near $1 / 10$ in magnetometry and slow-light applications $[9,13,14]$, where longer phase evolution in the dark makes line shapes more sensitive to magnetic fields or allows long-time-scale slow-light pulse delay. The geometry of the cell and beam is important in determining EIT line shapes in coated cells, as the size ratio sets the relative scales for average times $\tau_{\text {in }}$ and $\tau_{\text {out }}$ that the atoms spend inside and outside the laser beam. In this picture, a larger laser beam will reduce $\tau_{\text {out }}$ and increase $\tau_{\text {in }}$, leading to a narrower broad structure (which is based on beam transit time), but to a broader narrow structure.

We measured EIT resonances for a number of laser-beam sizes. For the broad structure [Fig. 5(a)], EIT linewidths scale like $\gamma_{\mathrm{EIT}}=\Omega_{c}^{2} / \gamma+\gamma_{0}$ (i.e., linearly with laser intensity), where $\gamma_{0}$ is the full coherence loss rate including losses due to 

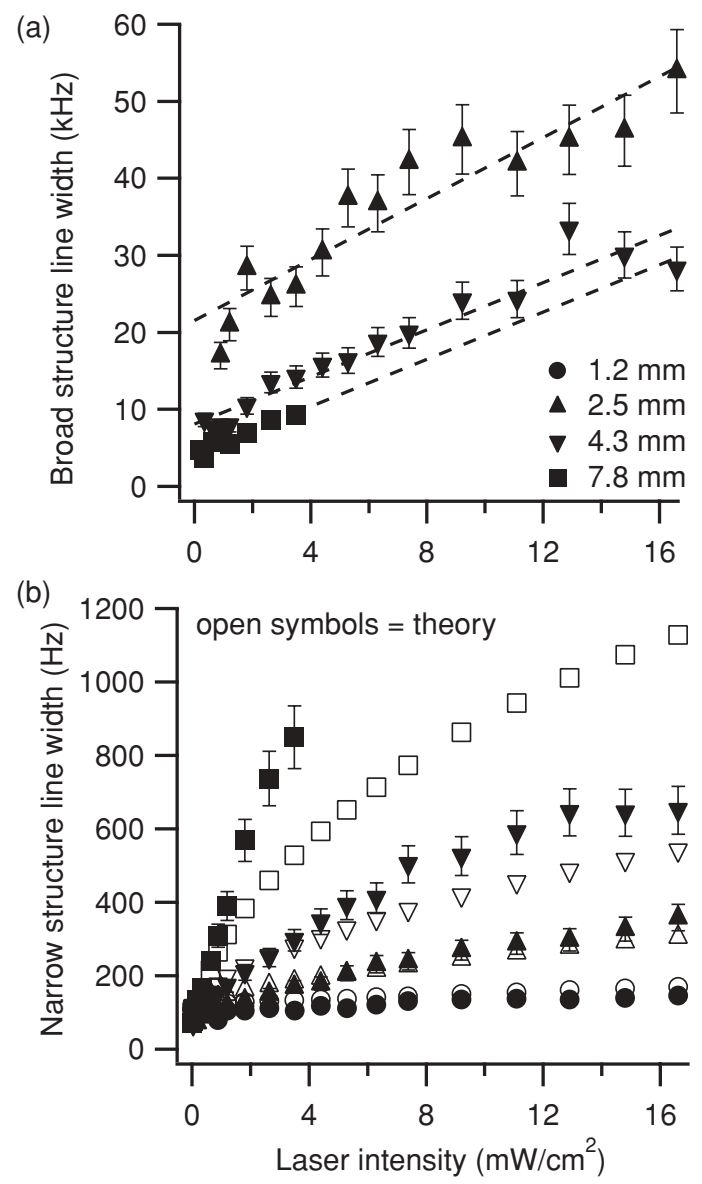

FIG. 5. EIT linewidths for a range of beam diameters at $T=$ $45{ }^{\circ} \mathrm{C}$. (a) The broad structure width is based on the average transit (bright) time $\tau_{\text {in }}$, and is thus smaller for large beams, including the non-power-broadened widths. (b) The narrow structure has the opposite behavior - the width is limited by the average dark time $\tau_{\text {out }}$ outside the beam, so larger beams result in wider line shapes. Open symbols are results from our theoretical model, which employs no free parameters. Disagreement with experimental measurements for large beams is due to the use of an exponential distribution for $t_{d}$. (See Appendix A).

atoms leaving the laser beam. For low control-field intensities, the first term is negligible and the width scales inversely with the transit time across the beam. Thus, smaller beams yield wider line shapes for any given power. This also occurs for line shapes in non-coated cells with buffer gas.

However, as expected, the narrow resonance linewidth has the opposite behavior [Fig. 5(b)]. Smaller beams allow longer phase evolution in the dark, making the EIT line shapes narrower. Results from our numerical simulations based on the Ramsey pulse sequence picture (described further in [32]) are also shown. At low intensity or small beam size, atoms require multiple trips through the beam before being optically pumped into the dark state. The equilibrium fraction of atoms found in the dark state is determined by many independent atom-field interactions and dark times during an optical pumping period. As the number of interactions increases, the choice of individual $t_{\text {in }}$ and $t_{\text {out }}$ distributions becomes less important. Also, the effective laser-beam size increases somewhat with intensity, reducing the time atoms spend in the dark. Hence, our numerical modeling results agree with experiment better for the smaller beam sizes and low intensities, where our approximate distribution is more accurate. The ultranarrow EIT width is highly sensitive to the distribution of dark times. The exact distribution is known, but we assumed an exponential distribution for $t_{d}$ with a mean equal to the mean of the exact distribution to make calculations tractable. This overestimates the number of atoms returning to the beam after a very short time, but quantitative predictions of linewidths are still possible for small beam sizes or low field intensities due to the final result being dominated by sums of many crossing times rather than by the evolution in a single crossing. See Appendix A for further discussion.

This opposing geometry dependence of the broad and narrow EIT structures is important for choosing beam and cell size in a given application. For small beams, the two structures are well defined and distinct (for extremely small beams, however, the narrow structure is not prominent); when using one of the linewidths, the presence of the other feature must be taken into account. For example, a slow- or stored-light experiment with a small beam could use the broad EIT structure (due to its higher bandwidth, and improved contrast from better optical pumping due to the coating), but the sharp, narrow feature would add distortion to signals sent into the medium. For a large beam approaching the cell size, the broad and narrow widths approach each other, and the two features are difficult to resolve, resulting in a non-Lorentzian EIT shape.

Magnetic field gradients will also affect the character of the two EIT structures, and this depends on the choice of laser-beam size. Such gradients will broaden the single-passbased broad structure, since atoms in different regions of the cell sample different magnetic fields (and thus different twophoton detuning); the narrow structure is much less affected because multiple beam passes at different $z$ positions lead to positional averaging [6]. Larger laser beams will improve the motional averaging, but also expose the atoms to potentially larger magnetic field gradients.

The EIT spectra used to generate the data in Figs. 4 and 5 were fit to the sum of two Lorentzians. Error bars associated with EIT linewidths were derived from: (i) statistical uncertainty in Lorentzian fits to the data, (ii) uncertainty in the laser frequency leading to systematic variation in the width of typically $\pm 10 \%$, and (iii) systematic uncertainty from difficulty distinguishing broad and narrow features. We varied the fitting range to estimate the uncertainty in fitting dual Lorentzians to our data. For larger beams and high laser intensity, the type (iii) error becomes large because the narrow and broad widths become similar and the narrow structure contrast diminishes.

\section{B. Coated-cell EIT contrasts}

EIT contrast indicates the strength of interaction between input light and atoms, and high contrast levels are preferred in most experimental applications. We investigated EIT contrast over more than three orders of magnitude in laser intensity, for a range of vapor-cell temperatures. Figure 6 shows the EIT contrast for both broad and narrow structures. Contrast for both structures increases from zero with laser intensity as the pumping rate becomes large compared to the ground-state 

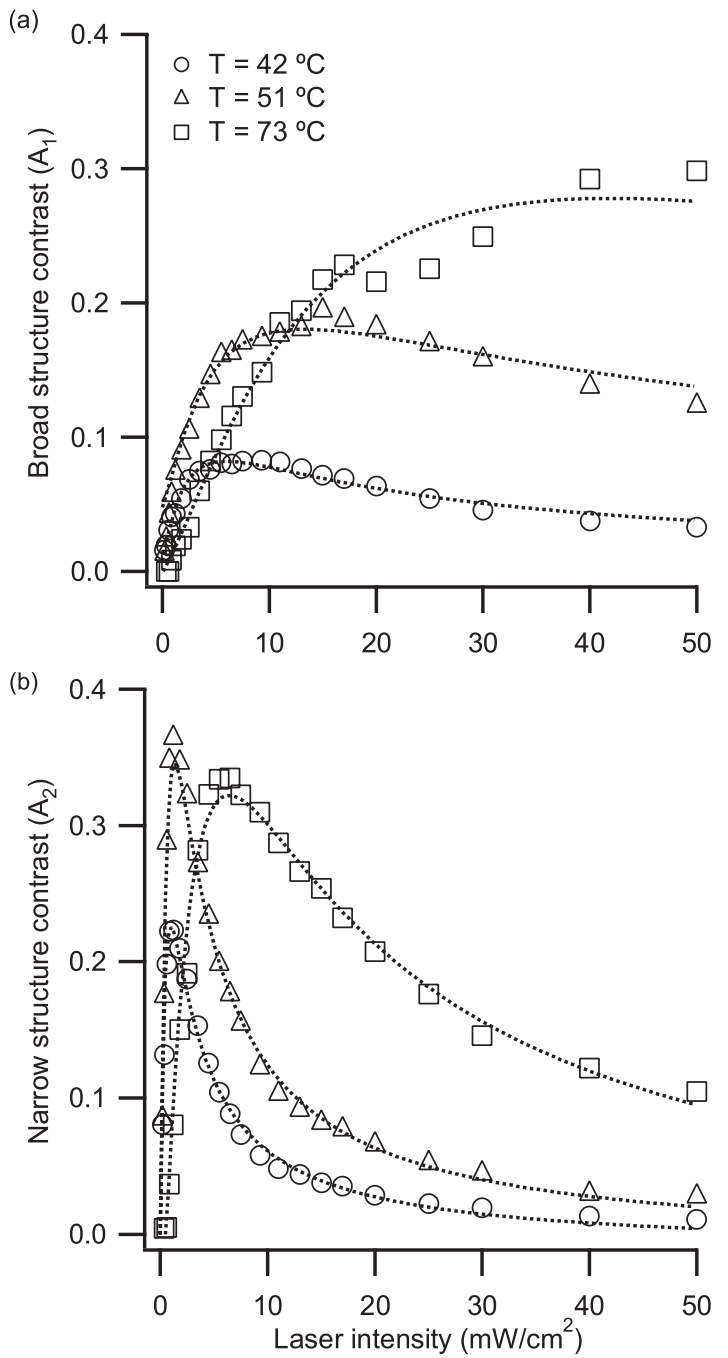

FIG. 6. EIT contrast versus laser intensity for $d=3 \mathrm{~mm}$ at various temperatures. (a) Broad-structure contrast. (b) Narrow-structure contrast. Peaks of these plots may indicate optimal conditions for experiments performed in coated cells. Dotted curves are fits to a four-level model (see text).

decoherence rate. At lower intensity, atoms lose coherence before being fully pumped into the dark state. At high intensity, contrast falls because the offset floor (off-resonant transmission) of the EIT increases. This occurs because of optical pumping into the trapped-state sublevels $\left|F, m_{F}\right\rangle=$ $|1,-1\rangle,|1,0\rangle$, and $|1,+1\rangle$, so fewer atoms participate in EIT. Contrast data was fit to a four-level EIT model based on [33], in which a standard $\Lambda$-scheme EIT with two input light fields was augmented with an additional ground state acting as a "trapped" state reached by excited-state decay and inaccessible to the light fields.

Despite the greater optical depth, peak contrast levels eventually fall off at high temperature [Fig. 6(b)], an effect we attribute to radiation trapping [13,34-36], where spontaneously emitted photons are reabsorbed by the atomic medium; atoms in the dark state that absorb such photons lose their coherence. This also explains why the peak contrast occurs at higher laser intensity for higher temperature, as a faster pumping rate into the dark state is required to reach maximal coupling. Contrast peaking at a transverse optical depth of $\sim 1$ or 2 in our operating regime is consistent with a simple three-level EIT analysis, with an appropriate radiation trapping term added to the ground-state decoherence rate [37]. It is also consistent with indirect measurements of radiation trapping in buffer gas cells [38] at atomic densities similar to those used here. We expect radiation trapping to be an even more significant effect in coated cells, since atoms throughout the cell volume participate in EIT, and thus spontaneously emitted photons reduce overall coherence along the entire path exiting the cell.

At temperatures beyond those investigated here, $\mathrm{Rb}-\mathrm{Rb}$ spin exchange collisions become important and interfere with favorable EIT conditions; the greater decoherence rate broadens the widths of both structures and leads to diminished contrast. In the present study, our highest temperature of $73{ }^{\circ} \mathrm{C}$ yields broadening due to spin exchange of approximately $160 \mathrm{~Hz}$ (based on the Rb-Rb cross section, atomic speed, and density), which is negligible for power-broadened situations, but potentially important for narrow-width applications including vapor-cell clocks and magnetometers. At our lowest temperature of $42{ }^{\circ} \mathrm{C}$, spin exchange broadening is below $20 \mathrm{~Hz}$, which would contribute significantly to overall linewidth only in systems with very high quality coating and magnetic field gradient reduction.

Four-wave mixing can also contribute to line broadening in vapor-cell EIT systems, but not significantly for Zeeman EIT with a weak probe field, which is unable to drive the fourwave-mixing cycling transition. In coated-cell EIT systems using hyperfine EIT, a strong control field is sufficient to see such effects.

Past work $[13,14]$ has shown that slow-light probe pulses can be delayed on either the broad- or narrow-structure time scale, even in intermediate intensity regimes when both structures are present. However, the extended spin coherence lifetime supported by the wall coating exists during the storage interval (when the input light is off) regardless of which EIT structure was used to slow and store the input pulse. Thus, one can find a maximal broad structure contrast level at high laser intensity, then send a corresponding highbandwidth probe pulse into the medium (with minimal narrow structure contrast, to avoid pulse distortion). The pulse can be stored as long as coherence is preserved, then read out. For example, values extracted from Figs. 5 and 6 indicate that a 3-mm diameter beam with $50 \mathrm{~mW} / \mathrm{cm}^{2}$ intensity sent into a $T=73{ }^{\circ} \mathrm{C}$ vapor cell would establish EIT with a dominant broad structure of $\sim 150 \mathrm{kHz}$; a bandwidth-matching $30-\mu \mathrm{s}$ slow-light pulse could be stored for several ms. This should lead to storage times much longer than the temporal pulse width, where we use the transit-time scale for writing, but the coherence-time scale for storage.

\section{CONCLUSIONS}

We have investigated characteristics of the dual-structured EIT line shape found in coated cells. Line-shape contrast and width are strongly affected by laser-beam intensity, cell temperature (atomic density), and cell-beam geometry. The ultranarrow feature for Zeeman EIT can have a high contrast when optimized with respect to these parameters. Contrast 
is likely limited, however, by off-resonant absorption $[39,40]$ and the resulting radiation trapping. Altering the aspect ratio of the cell is one promising avenue, as a reduced diameter would lead to less time spent in the cell by spontaneously emitted photons; and a longer cell could allow lower atomic densities for the same optical depth. The reduced contrast at high laser intensity could also be addressed with a second laser acting as a repumper to depopulate the trapped state.

Antirelaxation coated cells provide the possibility for longtime-scale experiments, including storing high-bandwidth pulses, and may enhance numerous applications. We have discussed their unique features, and used a model based on the times atoms spend inside and outside the beam to capture much of the EIT physics in these cells. Care must be taken in employing coated cells due to their characteristic dual-structured EIT shape, and geometry dependence and saturation conditions not seen in other types of vapor cells.

\section{APPENDIX A: THEORETICAL MODEL}

The evolution of the internal degrees of freedom of atoms traversing laser fields multiple times before decohering may be calculated by evaluating a distribution of Ramsey pulse sequences $[22,28]$. The steady-state EIT spectrum is determined by the average atomic state after a sequence of Ramsey pulses of varying duration, delays between pulses, and effective frequency due to factors such as the distribution of atomic velocities and the geometry of the system. To determine the steady-state density matrix for the atomic ensemble in the laser beam, we average over all appropriate paths.

Following a formalism that has been used to determine line shapes of buffer gas EIT resonances [22,23], we represent the atomic $\Lambda$ system with a Bloch vector [41]. Adiabatically eliminating the excited state of the $\Lambda$ system and expressing the ground states in the bright and dark basis yields an effective two-level system. The density matrix $\rho$ of this two-level system is represented with a Bloch vector $\mathbf{R}(t)$, which, for constant fields, evolves according to

$$
\frac{d}{d t}\left[\begin{array}{l}
R_{1} \\
R_{2} \\
R_{3}
\end{array}\right]=\left[\begin{array}{lll}
-\alpha^{\prime} & -\beta^{\prime} & 0 \\
\beta^{\prime} & -\alpha^{\prime} & S \delta \\
0 & -S \delta & -\alpha^{\prime}
\end{array}\right]\left[\begin{array}{l}
R_{1} \\
R_{2} \\
R_{3}
\end{array}\right]+\left[\begin{array}{l}
0 \\
0 \\
\alpha
\end{array}\right],
$$

where the dark-state population is $\rho_{d d}=\left(1+R_{3}\right) / 2$, the bright-state population is $\rho_{b b}=\left(1-R_{3}\right) / 2$, and the coherence between the bright and dark states is $\rho_{d b}=\left(R_{1}-i R_{2}\right) / 2$. The optical-pumping rate $\alpha$ and frequency shift $\beta$ are

$$
\alpha=\frac{|\Omega|^{2} \Gamma / 2}{\Gamma^{2}+4 \Delta^{2}}, \quad \beta=\frac{|\Omega|^{2} \Delta}{\Gamma^{2}+4 \Delta^{2}},
$$

where $\Gamma$ is the radiative relaxation rate of the excited state, $\Omega=$ $\sqrt{\left|\Omega_{C}\right|^{2}+\left|\Omega_{P}\right|^{2}}, S=\sin 2 \theta$ and $C=\cos 2 \theta$, where $\sin \theta=$ $\Omega_{P} / \Omega$. The damping rate of the Bloch vector $\alpha^{\prime}=\alpha+\gamma_{0}$, where $\gamma_{0}$ is the atomic ground-state decoherence rate including the effects of stray magnetic field gradients and residual dephasing due to wall collisions. Energy shifts associated with two-photon detunings are included via $\beta^{\prime}=\beta-C \delta$. One-photon detuning $\Delta$ is the offset of the laser from the ground to excited state transition and $\delta$, the two-photon detuning, is the difference between the frequencies of the two fields and the frequency difference of the ground states of the $\Lambda$ system (see Fig. 1). For constant fields, Eq. (A1) can be integrated to yield

$$
\mathbf{R}(t)=A(t) \mathbf{R}_{0}+[I-A(t)] \mathbf{R}_{s},
$$

where $\mathbf{R}_{0}$ is the Bloch vector at $t=0, \mathbf{R}_{s}$ is the steady-state solution of Eq. (A1), $A(t)$ is a $3 \times 3$ matrix encapsulating the time dependence [32], which can be found via Eq. (A1), and $I$ is the identity matrix. Note that out of the beam $R_{s}=0$ and thus $R_{0}=0$ for an unpolarized atom entering the beam. $A(t)$ may also be broken into two components $A_{\text {in }}(t)$ and $A_{\text {out }}(t)$ describing the internal state evolution in and out of the laser fields.

In averaging over all possible atomic paths, we assume that atomic external degrees of freedom completely thermalize upon contact with the wall, and that we may treat the selection of atoms' speeds and trajectories after each wall collision as the result of a Markovian process. The average state of an initially unpolarized atom $(\mathbf{R}=0)$ that has completed one passage across the beam and is about to re-enter the beam (after any number of cell crossings that missed the beam) is given by

$$
\left\langle\mathbf{R}_{1}\right\rangle=\left(\left\langle A_{\text {out }}\right\rangle-\left\langle A_{\text {out }} A_{\text {in }}\right\rangle\right) \mathbf{R}_{s} .
$$

The average $\left\langle A_{\text {out }} A_{\text {in }}\right\rangle$ is taken over the distribution of time spent in the beam and out of the beam before re-entering. We then approximate $\left\langle A_{\text {out }} A_{\text {in }}\right\rangle \simeq\left\langle A_{\text {out }}\right\rangle\left\langle A_{\text {in }}\right\rangle$. Treating the vapor cell as an infinite cylinder, the mean number of times an atom crosses a cell of diameter $D$ before encountering a laser beam of diameter $d$ is $(d / D)^{-1}$. Choosing $d / D \ll 1$, we may treat $\tau_{\text {out }}$ (the time spent out of the laser beam) as if it were independent of $\tau_{\text {in }}$ (the time spent crossing the beam). After $N$ passages,

$$
\left\langle\mathbf{R}_{N}\right\rangle=\left\langle A_{\text {out }}\right\rangle\left(\sum_{k=0}^{N-1}\left(\left\langle A_{\text {in }}\right\rangle\left\langle A_{\text {out }}\right\rangle\right)^{k}\right)\left(I-\left\langle A_{\text {in }}\right\rangle\right) \mathbf{R}_{s} .
$$

Taking $N \rightarrow \infty$, we obtain the steady-state distribution of the atomic ensemble.

Because the time-dependent terms of $A_{\text {in }}(t)$ and $A_{\text {out }}(t)$ are of the form $e^{-\gamma t} \sin \eta t$ and $e^{-\gamma t} \cos \eta t$, we need only find the form of $\left\langle e^{-(\gamma-i \eta) t}\right\rangle$ to determine the matrix averages. While the exact probability density function (pdf) governing the time spent in and out of the beam is difficult to evaluate [32], for small $d / D$, we approximate the pdf with an exponential:

$$
g_{\text {in,out }}(t)=\frac{\sqrt{\pi}}{\tau_{\text {in,out }} \sqrt{2}} e^{-\sqrt{\frac{\pi}{2}} t / \tau_{\text {in,out }}},
$$

where the mean times in and out of the beam, $\tau_{\text {in }}$ and $\tau_{\text {out }}$, have been rescaled by a factor of $\sqrt{2 / \pi}$ to agree with numerical evaluation of the exact pdf [32]. These approximations work best in the small-beam limit $(d / D \ll 1)$ and for weak fields, where the results are less sensitive to the form of the probability distribution of the interaction times, and more dependent upon the overall effective optical pumping rate as determined by the mean fraction of the time atoms spend in the beam. 


\section{A. Weak-field limit, no Doppler averaging}

In the absence of Doppler broadening, the steady-state Bloch vector may be evaluated explicitly. Assuming both the weak-probe field limit $\Omega_{P} \ll \Omega_{C}$ and zero one-photon detuning, $A_{\text {in }}(t)$ and $A_{\text {out }}(t)$ differ only by a factor of $e^{-\alpha t}$ as defined in Eq. (A2). Averaging over the exponential time distributions $g_{\text {in }}(t)$ and $g_{\text {out }}(t)$, we find the averaged time dependence in and out of the beam to be given by

$$
\begin{aligned}
& X(\delta)=\left\langle e^{-\left(\alpha+\gamma_{0}-i \delta\right) t}\right\rangle_{g_{\text {in }}}=\frac{\sqrt{\pi} /\left(\sqrt{2} \tau_{\text {in }}\right)}{\left[\sqrt{\pi} /\left(\sqrt{2} \tau_{\text {in }}\right)+\alpha+\gamma_{0}\right]-i \delta}, \\
& Y(\delta)=\left\langle e^{-\left(\gamma_{0}-i \delta\right) t}\right\rangle_{g_{\text {out }}}=\frac{\sqrt{\pi} /\left(\sqrt{2} \tau_{\text {out }}\right)}{\left[\sqrt{\pi} /\left(\sqrt{2} \tau_{\text {out }}\right)+\gamma_{0}\right]-i \delta} .
\end{aligned}
$$

Setting

$$
Z(\delta)=\sum_{n=0}^{\infty}[X(\delta) Y(\delta)]^{n}[1-X(\delta)]=\frac{1-X(\delta)}{1-X(\delta) Y(\delta)},
$$

the steady-state Bloch vector is

$$
\begin{aligned}
\langle\mathbf{R}\rangle= & {\left[\begin{array}{lcl}
C^{2} \operatorname{Re} Z(\delta) & C \operatorname{Im} Z(\delta) & -S C \operatorname{Re} Z(\delta) \\
-C \operatorname{Im} Z(\delta) & \operatorname{Re} Z(\delta) & S \operatorname{Im} Z(\delta) \\
-S C \operatorname{Re} Z(\delta) & -S \operatorname{Im} Z(\delta) & S^{2} \operatorname{Re} Z(\delta)
\end{array}\right] \mathbf{R}_{s} } \\
& +\left[\begin{array}{ccc}
S^{2} Z(0) & 0 & S C Z(0) \\
0 & 0 & 0 \\
S C Z(0) & 0 & C^{2} Z(0)
\end{array}\right] \mathbf{R}_{s} .
\end{aligned}
$$

Figure 7 shows EIT spectra determined by evaluating Eq. (A10) and extracting the optical coherence [41] for a variety of beam diameters. The ultranarrow transmission feature of the EIT spectrum is due to atomic coherence accumulating a relative phase in the dark. Increasing the diameter of the beam relative to the cell reduces the amount of time atoms spend in the dark, and thus reduces the contrast of the Ramsey-narrowed transmission peak.

\section{B. Doppler broadening}

In thermal vapor cells, one-photon transitions are Doppler broadened. Doppler broadening leads to EIT transmission peaks narrower than those of Sec. A1 due to reduced coupling to the optical fields, reducing the overall interaction and increasing the lifetime of the atomic coherence in the powerbroadened limit.

In the presence of Doppler broadening, the Bloch vector $\mathbf{R}$ is composed of many sub-ensembles of atoms with different axial velocities $v$ and different couplings to the applied fields. The Bloch vector $\mathbf{R}(v)$ undergoes three different processes: interaction with the beam, evolution outside the beam, and interaction with the wall.

Evolution of the Bloch vector, $\mathbf{R}(v)$, in the presence of Doppler broadening is identical to Eq. (A3) except that the time evolution matrix also depends upon velocity:

$$
A_{\text {in,out }}(t) \rightarrow A_{\text {in,out }}(t, v) .
$$

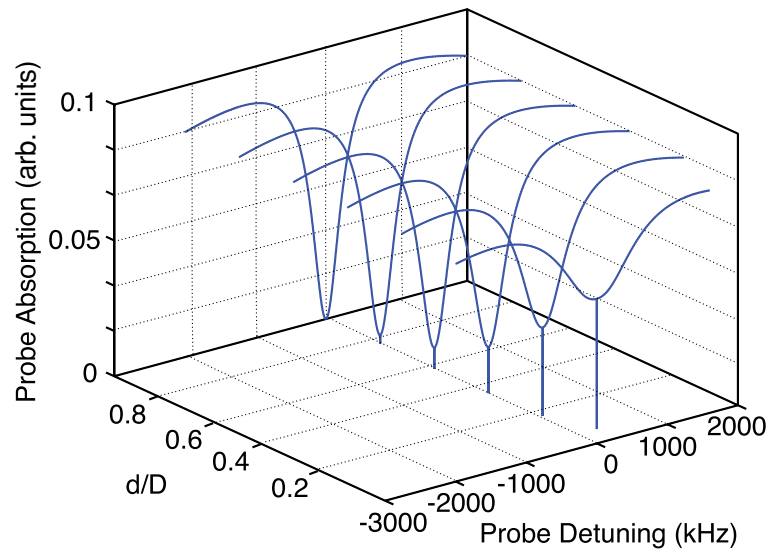

FIG. 7. (Color online) Probe absorption as a function of probe detuning, for beams with equal intensity, but where the diameter ratio $d / D$ varies between 0.01 and 1 . For small beam diameters, the EIT spectrum is well described by a model with ground-state decoherence determined by the finite atomic transit time, except in the neighborhood of two-photon resonance, where the Ramsey-narrowed absorption minima resides. As the beam diameter increases, more and more atoms in the beam at any given time are pumped into the dark state, decreasing the contrast of the narrowed feature, until all atoms spend all their time in the beam, and the ground-state lifetime is limited by the coating and power broadening. These calculations were performed for typical experimental parameters: cell diameter $D=2.54 \mathrm{~cm}$ and atomic velocities corresponding to $T=43{ }^{\circ} \mathrm{C}$.

The velocity dependence shifts the effective one-photon detuning, leading to velocity dependence of pumping rates, decoherence rates, and energy shifts. Interaction with the cell coating rethermalizes the atoms, yielding the output distribution

$$
\mathbf{R}_{\text {out }}(v)=\int_{-\infty}^{\infty} d v^{\prime} f\left(v^{\prime}\right) \mathbf{R}_{\mathrm{in}}\left(v^{\prime}\right),
$$

where $f\left(v^{\prime}\right)$ is the Maxwell-Boltzman distribution for the axial velocity of the vapor. Two applications of Eq. (A12) are equivalent to one application, and evolution of $\mathbf{R}(t, v)$ via Eq. (A3) commutes with the rethermalization of Eq. (A12). Thus, we model the sequence of evolution in the dark, followed by collision with the cell coating, followed by further evolution in the dark, by first applying Eq. (A12) and then Eq. (A3) with appropriate shifts of $\Delta$, the one-photon detuning, for each velocity class.

Depolarized atoms are represented by $\mathbf{R}=\mathbf{0}$ regardless of their velocity class, so we may drop the velocity dependence from $\mathbf{R}_{\text {in }}$ since atoms never re-enter the beam without first rethermalizing on the cell coating. In light of these considerations, we may take the average over all axial velocities immediately upon atoms' exiting the beam. The Bloch vector after interaction for a time $t_{\text {in }}$ in the beam is given by

$$
\mathbf{R}\left(t_{\text {in }}\right)=B\left(t_{\text {in }}\right) \mathbf{R}_{\text {in }}+\mathbf{C}\left(t_{\text {in }}\right),
$$

where

$$
\begin{gathered}
B\left(t_{\mathrm{in}}\right)=\int_{-\infty}^{\infty} d v f(v) A_{\text {in }}\left(t_{\text {in }}, v\right) \\
\mathbf{C}\left(t_{\text {in }}\right)=\int_{-\infty}^{\infty} d v f(v)\left[\mathbf{R}_{s}(v)-A_{\text {in }}\left(t_{\text {in }}, v\right) \mathbf{R}_{s}(v)\right] .
\end{gathered}
$$


Evolution in the dark is as simple as it was in Sec. A1, as $\mathbf{R}\left(t_{\text {out }}\right)=A_{\text {out }}\left(t_{\text {out }}\right) \mathbf{R}_{\text {in }}$. We now take the averages over $t_{\text {in }}$ and $t_{\text {out }}$ to obtain

$$
\begin{gathered}
\langle A\rangle=\int_{0}^{\infty} d t \frac{\sqrt{\pi} e^{-\sqrt{\frac{\pi}{2}} \frac{t}{\tau_{\text {out }}}}}{\sqrt{2} \tau_{\text {out }}} A_{\text {out }}(t), \\
\langle B\rangle=\int_{0}^{\infty} d t \frac{\sqrt{\pi} e^{-\sqrt{\frac{\pi}{2}} \frac{t}{\tau_{\text {in }}}}}{\sqrt{2} \tau_{\text {in }}} B(t), \\
\langle\mathbf{C}\rangle=\int_{0}^{\infty} d t \frac{\sqrt{\pi} e^{-\sqrt{\frac{\pi}{2}} \frac{t}{\tau_{\text {in }}}}}{\sqrt{2} \tau_{\text {in }}} \mathbf{C}(t) .
\end{gathered}
$$

The Doppler-averaged steady-state Bloch vector immediately prior to interaction with the beam is then given by

$$
\mathbf{R}_{s s}=\langle A\rangle \sum_{n=0}^{\infty}(\langle B\rangle\langle A\rangle)^{n}\langle\mathbf{C}\rangle,
$$

which can be written in closed form by finding the eigenvector matrix $P$ and the eigenvalues $\lambda_{1,2,3}$ of $\langle B\rangle\langle A\rangle$ as

$$
\mathbf{R}_{s s}=\langle A\rangle P\left[\begin{array}{ccc}
\frac{1}{1-\lambda_{1}} & 0 & 0 \\
0 & \frac{1}{1-\lambda_{2}} & 0 \\
0 & 0 & \frac{1}{1-\lambda_{3}}
\end{array}\right] P^{-1}\langle\mathbf{C}\rangle .
$$

Finally, to determine the equilibrium distribution $\mathbf{R}(v)$ in the beam, we apply

$$
\begin{aligned}
\mathbf{R}(v) & =\int_{0}^{\infty} d t_{\text {in }} \frac{d}{2} e^{-t d / 2} A_{\text {in }}\left(t_{\text {in }}, v\right) \mathbf{R}_{s s} \\
& +\left[I-\int_{0}^{\infty} d t_{\text {in }} \frac{d}{2} e^{-t d / 2} A_{\text {in }}\left(t_{\text {in }}, v\right)\right] \mathbf{R}_{s}(v),
\end{aligned}
$$

where $d$ is the laser-beam diameter and $I$ is the identity matrix. The measured optical coherence is then given by averaging over $v$ :

$$
\begin{aligned}
\rho_{c, a}= & \int_{-\infty}^{\infty} d v \frac{e^{-\frac{v^{2}}{2 \sigma_{v}}}}{\sigma_{v} \sqrt{2 \pi}}\left[\frac{i \alpha(v)-\beta(v)}{\Omega^{2}}\right] \\
& \times\left\{\Omega_{P}\left[1-\mathbf{R}_{\mathbf{3}}(v)\right]-\Omega_{C}\left[\mathbf{R}_{\mathbf{1}}(v)-i \mathbf{R}_{\mathbf{2}}(v)\right]\right\} .
\end{aligned}
$$

Equations (A15) and (A17) can be evaluated numerically, and Fig. 5 shows results of this calculation compared to measured EIT resonances. Note that, although we have good quantitative agreement for the smallest beam diameters, our calculated Ramsey-narrowed bandwidths deviate from the experimental results as the beam diameter and the applied field intensities increase. This remaining discrepancy results from our choosing to approximate the beam interaction time distribution with an exponential. While the exponential distribution has nice algebraic properties, it reaches a maximum at $t=0$, while the actual time distribution's pdf falls to zero at $t=0$. This causes our model to underestimate the time atoms spend interacting with the beam, and thus we overestimate the effective lifetime of the atomic coherence. Longer coherence times result in narrower EIT bandwidths. This artifact becomes more pronounced as the beam size is increased. Although our limited experimental laser power prevented us from fully saturating the Ramsey-narrowed transmission peak for any but the smallest beams, we expect our model to show better agreement with experiment at higher intensities, so long as the beams do not become so large as to produce significant correlations between time spent in the dark and time spent in the beam.
[1] S. E. Harris, Phys. Today 50, 36 (1997).

[2] E. Arimondo, Phys. Rev. A 54, 2216 (1996).

[3] M. A. Bouchiat and J. Brossel, Phys. Rev. 147, 41 (1966).

[4] H. G. Robinson and C. E. Johnson, Appl. Phys. Lett. 40, 771 (1982).

[5] H. G. Robinson, E. S. Ensberg, and H. G. Dehmelt, Bull. Am. Phys. Soc. 3, 9 (1958).

[6] H. M. Goldenberg, D. Kleppner, and N. F. Ramsey, Phys. Rev. Lett. 5, 361 (1960).

[7] D. Budker, V. V. Yashchuk, and M. Zolotorev, Phys. Rev. Lett. 81, 5788 (1998).

[8] M. T. Graf, D. F. Kimball, S. M. Rochester, K. Kerner, C. Wong, D. Budker, E. B. Alexandrov, M. V. Balabas, and V. V. Yashchuk, Phys. Rev. A 72, 023401 (2005).

[9] D. Budker, W. Gawlik, D. F. Kimball, S. M. Rochester, V. V. Yashchuk, and A. Weis, Rev. Mod. Phys. 74, 1153 (2002).

[10] A. Kuzmich, L. Mandel, and N. P. Bigelow, Phys. Rev. Lett. 85, 1594 (2000).

[11] B. Julsgaard, A. Kozhekin, and E. S. Polzik, Nature (London) 413, 400 (2001).

[12] D. Budker, D. F. Kimball, S. M. Rochester, and V. V. Yashchuk, Phys. Rev. Lett. 83, 1767 (1999).

[13] M. Klein, I. Novikova, D. F. Phillips, and R. L. Walsworth, J. Mod. Opt. 53, 2583 (2006).
[14] Y. Xiao, M. Klein, M. Hohensee, L. Jiang, D. F. Phillips, M. D. Lukin, and R. L. Walsworth, Phys. Rev. Lett. 101, 043601 (2008).

[15] R. H. Dicke, Phys. Rev. 93, 99 (1954).

[16] R. W. Boyd and D. J. Gauthier, Prog. Optics 43, 497 (2002).

[17] L. V. Hau, S. E. Harris, Z. Dutton, and C. H. Behroozi, Nature (London) 397, 594 (1999).

[18] M. M. Kash, V. A. Sautenkov, A. S. Zibrov, L. Hollberg, G. R. Welch, M. D. Lukin, Y. Rostovtsev, E. S. Fry, and M. O. Scully, Phys. Rev. Lett. 82, 5229 (1999).

[19] C. Liu, Z. Dutton, C. H. Behroozi, and L. V. Hau, Nature (London) 409, 490 (2001).

[20] D. F. Phillips, A. Fleischhauer, A. Mair, R. L. Walsworth, and M. D. Lukin, Phys. Rev. Lett. 86, 783 (2001).

[21] A. S. Zibrov and A. B. Matsko, Phys. Rev. A 65, 013814 (2001).

[22] Y. Xiao, I. Novikova, D. F. Phillips, and R. L. Walsworth, Phys. Rev. Lett. 96, 043601 (2006).

[23] Y. Xiao, I. Novikova, D. F. Phillips, and R. L. Walsworth, Opt. Express 16, 14128 (2008).

[24] N. F. Ramsey, Phys. Rev. 78, 695 (1950).

[25] N. F. Ramsey, Molecular Beams (Clarendon, Oxford, 1956). 
[26] M. Fleischhauer and M. D. Lukin, Phys. Rev. Lett. 84, 5094 (2000).

[27] M. Fleischhauer and M. D. Lukin, Phys. Rev. A 65, 022314 (2002).

[28] S. I. Kanorsky, A. Weis, and J. Skalla, Appl. Phys. B: Lasers Opt. 60, S165 (1995).

[29] A. I. Okunevich, Opt. Spectrosc. 97, 834 (2004).

[30] A. I. Okunevich, J. Opt. Soc. Am. B 22, 29 (2005).

[31] M. Klein, M. Hohensee, Y. Xiao, R. Kalra, D. F. Phillips, and R. L. Walsworth, Phys. Rev. A 79, 053833 (2009).

[32] M. Hohensee, Ph.D. thesis, Harvard University, 2009.

[33] J. Vanier, M. W. Levine, D. Janssen, and M. Delaney, Phys. Rev. A 67, 065801 (2003).

[34] A. F. Molisch, B. P. Oehry, W. Schupita, and G. Magerl, J. Phys. B 30, 1879 (1997).
[35] A. B. Matsko, I. Novikova, M. O. Scully, and G. R. Welch, Phys. Rev. Lett. 87, 133601 (2001).

[36] A. B. Matsko, I. Novikova, and G. R. Welch, J. Mod. Opt. 49, 367 (2002).

[37] M. A. Rosenberry, J. P. Reyes, D. Tupa, and T. J. Gay, Phys. Rev. A 75, 023401 (2007).

[38] M. Klein, Y. Xiao, A. V. Gorshkov, M. Hohensee, C. D. Leung, M. R. Browning, D. F. Phillips, I. Novikova, and R. L. Walsworth, Proc. SPIE 6904, 69040C (2008).

[39] R. W. Boyd, D. J. Gauthier, A. L. Gaeta, and A. E. Willner, Phys. Rev. A 71, 023801 (2005).

[40] A. B. Matsko, D. V. Strekalov, and L. Maleki, Opt. Express 13, 2210 (2005).

[41] S. M. Shahriar, P. R. Hemmer, D. P. Katz, A. Lee, and M. G. Prentiss, Phys. Rev. A 55, 2272 (1997). 